\title{
The loss of function of $H E L$, which encodes a cellulose synthase interactive protein, causes helical and vine-like growth of tomato
}

\author{
Qihong Yang ${ }^{1}$, Xiaoshuai Wan', Jiaying Wang ${ }^{1}$, Yuyang Zhang $\mathbb{1}^{1}$, Junhong Zhang ${ }^{1}$, Taotao Wang ${ }^{1}$, \\ Changxian Yang ${ }^{1}$ and Zhibiao Ye
}

\begin{abstract}
Helical growth is an economical way for plant to obtain resources. The classic microtubule-microfibril alignment model of Arabidopsis helical growth involves restriction of the appropriate orientation of cellulose microfibrils appropriately in the cell walls. However, the molecular mechanism underlying tomato helical growth remains unknown. Here, we identified a spontaneous tomato helical (hel) mutant with right-handed helical cotyledons and petals but left-handed helical stems and true leaves. Genetic analysis revealed that the hel phenotype was controlled by a single recessive gene. Using map-based cloning, we cloned the HEL gene, which encodes a cellulose interacting protein homologous to CSI1 of Arabidopsis. We identified a $27 \mathrm{bp}$ fragment replacement that generated a premature stop codon. Transgenic experiments showed that the helical growth phenotype could be restored by the allele of this gene from wild-type Pyriforme. In contrast, the knockout mutation of HEL in Pyriforme via CRISPR/Cas9 resulted in helical growth. These findings shed light on the molecular control of the helical growth of tomato.
\end{abstract}

\section{Introduction}

The rational use of growing space is essential for plant photosynthesis and reproductive development and indirectly affects biotic and abiotic resistance. Stretched leaves, upright growth, and deep roots enable more solar radiation and $\mathrm{CO}_{2}$ to be absorbed, thereby minimizing intraplant competition, to achieve increased photosynthesis efficiency ${ }^{1}$. A growth axis is usually generated in plants due to the expansion of organs into space, and a vast majority of plants display linear or circumferential growth $^{2}$. Notably, due to the lack of strong xylem support, some plants evolve a helical growth axis to achieve increased heights by winding onto a support, as observed for climbing plants. Specific organs of climbing plants, such as tendrils in Vitis, Pisum sativum, and

\footnotetext{
Correspondence: Changxian Yang (yangcx0915@mail.hzau.edu.cn) or Zhibiao Ye (zbye@mail.hzau.edu.cn)

${ }^{1}$ Key Laboratory of Horticultural Plant Biology, Ministry of Education, Huazhong Agricultural University, Wuhan 430070, China
}

These authors contributed equally: Qihong Yang and Xiaoshuai Wan.
Cucurbitaceae, often exhibit helical growth ${ }^{3}$. In addition, members of the legume family and other vine plants climb through stem circumnutation, the vast majority of which display a right-handed helix ${ }^{4}$. For some nonclimbing plants, pruning and bundling are essential for improving photosynthesis efficiency, and then yield and quality in agriculture.

Helical growth direction can be described as left- or righthandedness ${ }^{4}$. The left-handed helix involves clockwise rotation, as viewed from above. In contrast, right-handedness involves rotation in an anticlockwise direction. This phenomenon in climbing plants was described by Darwin a century ago. However, the molecular mechanisms underlying helical growth have been revealed only in the past two decades. Two loss-of-function mutations in Arabidopsis, SPIRAL 1 (SPR1) and SPIRAL 2 (SPR2), were first discovered to be responsible for righthanded helical growth ${ }^{5}$. Between them, the former caused right-handed helical growth of roots, stems, and hypocotyls, whereas the latter even extended to leaf petioles and petals.

\section{(c) The Author(s) 2020}

(c) (i) Open Access This article is licensed under a Creative Commons Attribution 4.0 International License, which permits use, sharing, adaptation, distribution and reproduction cc) in any medium or format, as long as you give appropriate credit to the original author(s) and the source, provide a link to the Creative Commons license, and indicate if changes were made. The images or other third party material in this article are included in the article's Creative Commons license, unless indicated otherwise in a credit line to the material. If material is not included in the article's Creative Commons license and your intended use is not permitted by statutory regulation or exceeds the permitted use, you will need to obtain permission directly from the copyright holder. To view a copy of this license, visit http://creativecommons.org/licenses/by/4.0/. 
The spr1/spr2 double mutant exhibited a more obvious phenotype than did each single mutant ${ }^{5}$. In addition, mutations in TUA4 and TUA6 which separately encode $\alpha$ tubulin 4 and $\alpha$-tubulin 6 resulted in the left-handed twisting of roots, cotyledons, leaves, and flowers ${ }^{6}$. Previous studies suggested that the cytoskeleton is closely related to helical growth of Arabidopsis ${ }^{5}$. Cortical microtubule (CMT) arrays have been shown to be oriented opposite to that of spiral growth of epidermal cell files ${ }^{6}$. SPR1, a microtubuleassociated protein (MAP) that localizes to the plus-end of microtubules (MTs), can stabilize the growing ends of CMTs and influence their dynamic properties ${ }^{7,8}$. Another plant-specific MAP, SPR2, is involved in the modulation of MT severity and regulates both the orientation of CMTs and the direction of organ growth ${ }^{9,10}$. Moreover, a series of mutations in tubulins or MAPs led to left- or right-handed helical growth of Arabidopsis, suggesting that various genetic mutations can cause twisted-type growth of several directions ${ }^{11-13}$.

CMTs, which are organized in arrays parallel to the central axis of cell expansion, inside the plasma membrane play an important role in controlling cell wall status and determining final cell shape ${ }^{14}$. The elongation or expansion of plant cells is restricted by the cell wall. Cellulose microfibrils (CMFs), which are synthesized by the cellulose synthase complex (CSC), act as the main factors linking CMTs and the cell wall during anisotropic growth of Arabidopsis ${ }^{15-18}$. Moreover, cellulose synthase interactive protein 1 (CSI1) functions as an interacting factor between CSCs and $\mathrm{CMTs}^{18-21}$. Knockout of Arabidopsis CSI1 caused left-handed helical growth of nearly all elongating organs, except for inflorescence stems, which exhibited right-handed twisting $^{20,21}$. CSI1 binds to both CSCs and CMTs during cell wall formation and acts as a tow cable involved in the movement of cellulose synthase rosettes ${ }^{19,21}$. Therefore, CSI1 constitutes a critical part of the microtubule-microfibril alignment model.

Mutational cytoskeletal-related genes in Arabidopsis provide cellular and genetic evidence that help us understand helical growth. The vegetative shoot apical meristem in Arabidopsis gives rise to leaves until inflorescence development ${ }^{22}$. The determinate growth habit of Arabidopsis is distinct from that of indeterminate plant species, such as tomato. Vegetative and reproductive phases of tomato regularly alternate, which results in an indeterminate plant ${ }^{23}$. Therefore, the suitability of microtubulemediated guidance during cellulose microfibril pattern formation in indeterminate tomato cultivars is still unclear. As observed for this type of tomato cultivar, cultivation requires plant supports such as garden stakes and twines to keep the tomato plant in an upright state.

In this study, we identified the $H E L$ gene, which encodes a cellulose synthase-interactive protein, via map-based cloning. Loss of function of the HEL gene caused righthanded helical growth of cotyledons and petals, whereas the opposite direction of stems and true leaves was observed in tomato. These results suggest that the function of $H E L$ is critical for helical growth of tomato, which provides a foundation for understanding the helical architectures of tomato.

\section{Materials and methods \\ Plant materials and mapping populations}

We identified a spontaneous mutant that exhibits a helical growth phenotype in the background of Pyriforme (Solanum lycopersicum var. cerasiforme), which is hereafter referred to as the hel mutant. We developed an $\mathrm{F}_{2}$ segregating population by crossing the hel mutant with LA1589 (Solanum pimpinellifolium). The hel mutant and Pyriforme were used as recipients for genetic transformation. All materials were grown in a greenhouse under standard conditions for phenotypic analysis. The height of the hel mutants and Pyriforme plants was measured once a week for a month. Eighteen tomato fruits were collected from three plants (6 fruits per plant), and their weight and fruit shape index were measured. In addition, the fruit set was measured after fruit enlargement. Three biological replicates were included.

\section{Extraction of RNA for RNA-seq}

Thirty wild-type and mutant $F_{2}$ individuals were collected and separately pooled as the N-pool and M-pool, respectively, based on their phenotypes. Total RNA was extracted using TRIzol reagent (Invitrogen, USA). After treatment with RNase-free DNase I (Takara, Japan), the RNA quantity and quality were then tested by using a NanoDrop 2000 instrument (Thermo Scientific, USA). RNA-seq libraries were generated using a NEBNext Ultra II RNA Library Prep Kit for Illumina (New England Biolabs, USA), according to the manufacturer's protocol. The libraries were subsequently sequenced on an Illumina HiSeq2000 platform (Novogene, China).

\section{BSR-seq mapping of HEL}

Raw reads from the RNA-seq experiment were scanned for quality and trimmed to remove low-quality bases. The clean reads from the N-pool and M-pool were mapped to the reference genome (Heinz1706) using BWA software (Availability: http://bio-bwa.sourceforge.net) ${ }^{24}$. SNPs were further identified and quantified at each SNP site in both pools using SAM software (Availability: http://biobwa.sourceforge.net) ${ }^{24}$. BSR-seq analysis was performed to determine the chromosomal location of $H E L$ according to the method described by Liu et al. ${ }^{25}$. Because both parents are homozygous lines, only homozygous SNPs were retained in both parents. The parameters of the SNP index were calculated to identify candidate regions for 
$H E L$. The average SNP index for a given genomic interval was estimated using a sliding window approach, with a $1 \mathrm{Mb}$ window size and $10 \mathrm{~kb}$ increment. A SNP index graph was subsequently constructed and plotted.

\section{DNA extraction and molecular marker generation}

Genomic DNA was isolated from young leaves of 4week-old tomato seedlings according to the method described by Fulton et al. ${ }^{26}$. Based on the nucleotide polymorphism identified from the hel mutant and LA1589 in the target region, three types of molecular markers were developed: InDel, SNP, and CAPS markers. All the primers used are listed in Table S1.

\section{Genotyping of $F_{2}$ individuals}

Genotyping of $F_{2}$ individuals was performed via PCR using polymorphic molecular markers. The PCR mixture consisted of $1 \mu \mathrm{L}$ of DNA, $2 \mu \mathrm{L}$ of $10 \times$ PCR buffer, $0.4 \mu \mathrm{L}$ of dNTPs $(10 \mathrm{mM}), 0.2 \mu \mathrm{L}$ of $2.5 \mathrm{U} / \mu \mathrm{L}$ Taq DNA polymerase (Invitrogen, USA), $0.4 \mu \mathrm{L}$ of each primer $(10 \mathrm{pm} /$ $\mu \mathrm{L}$ ), and $15.6 \mu \mathrm{L}$ of distilled water. The reaction parameters are described as follows: initial denaturation at $94{ }^{\circ} \mathrm{C}$ for $3 \mathrm{~min} ; 35$ cycles at $94^{\circ} \mathrm{C}$ for $30 \mathrm{~s}, 56^{\circ} \mathrm{C}$ for $30 \mathrm{~s}$, and $72{ }^{\circ} \mathrm{C}$ for $1 \mathrm{~min}$; and a final extension step at $72^{\circ} \mathrm{C}$ for $10 \mathrm{~min}$. The PCR products were then analyzed with a $1.5 \%$ $(w / v)$ agarose gel. For CAPS markers, the amplification products were further digested with appropriate restriction enzymes and then analyzed with a $1.5 \%(\mathrm{w} / \mathrm{v})$ agarose gel. The PCR-based SNP markers were genotyped via Sanger sequencing.

\section{Genetic mapping}

For all the polymorphic markers between the hel mutant and LA1589, $108 \mathrm{~F}_{2}$ recessive individuals were initially used for rough mapping of the $H E L$ gene. According to the rough mapping of $H E L$, new molecular markers were developed. Recombinant events were within a population of 925 individuals further screened using newly developed markers. The Genotypic and phenotypic data of $F_{2}$ recessive individuals were adopted for linkage analysis using the MapMaker/EXP 3.0 program ${ }^{27}$. The genetic distance was computed using the Kosambi mapping function.

\section{Gene prediction and sequence analysis}

The candidate genes in the target region between two closely linked markers were downloaded from the SGN database (ftp://sgn.cornell.edu). These genes were further analyzed using FGENESH and GeneScan. The primers used were designed based on the sequence information of these candidates using Primer 5.0. We amplified and sequenced the genomic and cDNA sequences of these candidates from the hel mutant and Pyriforme. The sequences were aligned with ClustalW2 and edited with
GeneDoc. All the primers used are listed in Supplementary Table S2.

\section{Vector construction and plant transformation}

The full-length coding sequence of ORF2 was cloned into pHellsgate 8 driven by the CaMV $35 \mathrm{~S}$ promoter. For CRISPR/Cas9 vector construction, we selected two adjacent target sites separated by a $340 \mathrm{bp}$ spacer in this candidate. These two constructs were separately transformed into hel mutants and Pyriforme via Agrobacterium tumefaciens strain GV $3101^{28}$. The resulting transgenic plants were screened with gene-specific primers.

\section{Results}

Phenotypic analyses of the hel mutant

We visually examined the helical growth phenotypes of the hel mutant. We found that the cotyledons, petals and pistils of the hel mutant showed right-handed helical growth (Fig. 1a-f). Compared with wild type, the hel mutant seedlings showed a lagging growth momentum at an early stage (Fig. $1 \mathrm{~g}$ and Supplementary Fig. S1a). Interestingly, the stems showed no helical growth at this stage. However, the growth direction of hel stems gradually shifted to left-handedness after flowering (Supplementary Fig. S1a-c). Similarly, the right-handed growth direction of the true leaves gradually shifted to left-handed twisting between the 3rd and 6th true leaves (Fig. 1a, b and Supplementary Fig. S1a). The hel phenotypes of the sepals, anther cones and inflorescence axis exhibited no obvious changes in comparison with those of the wild type (Fig. 1i-l and Supplementary Fig. S1d-g). Notably, the pistil length and fruit set of the hel mutant were significantly reduced (Fig. 1e, f, h). Moreover, sizes of the flowers and fruits showed no significant changes (Fig. 1c, d, j, l).

To detect the possible cytological changes underlying the helical growth of the hel mutant, we conducted cytological observations of the petiolules and rachises of compound leaflets using the paraffin sectioning technique. We found that the basal cells of petiolules were vertically arranged in wild type (Fig. 2a, c) but twisted in the hel mutant (Fig. 2b, d). Moreover, the cell arrangement exhibited a parallel arrangement in the wild-type rachis, whereas it was distorted in the rachis of the hel mutants (Fig. 2e, f).

We generated an $F_{2}$ segregating population from hel mutant $\times$ LA1589 cross. The $\mathrm{F}_{2}$ individuals comprised 344 wild-type and 108 helical plants, consistent with a 3:1 mendelian ratio $\left(\chi^{2}=0.103, P>0.05\right)$. In combination with the normal phenotype of $F_{1}$ plants, we inferred that the helical phenotype was controlled by a recessive gene.

\section{Map-based cloning of the HEL gene}

To determine the chromosomal location of the HEL gene, the BSR-seq method was performed using RNA-seq 


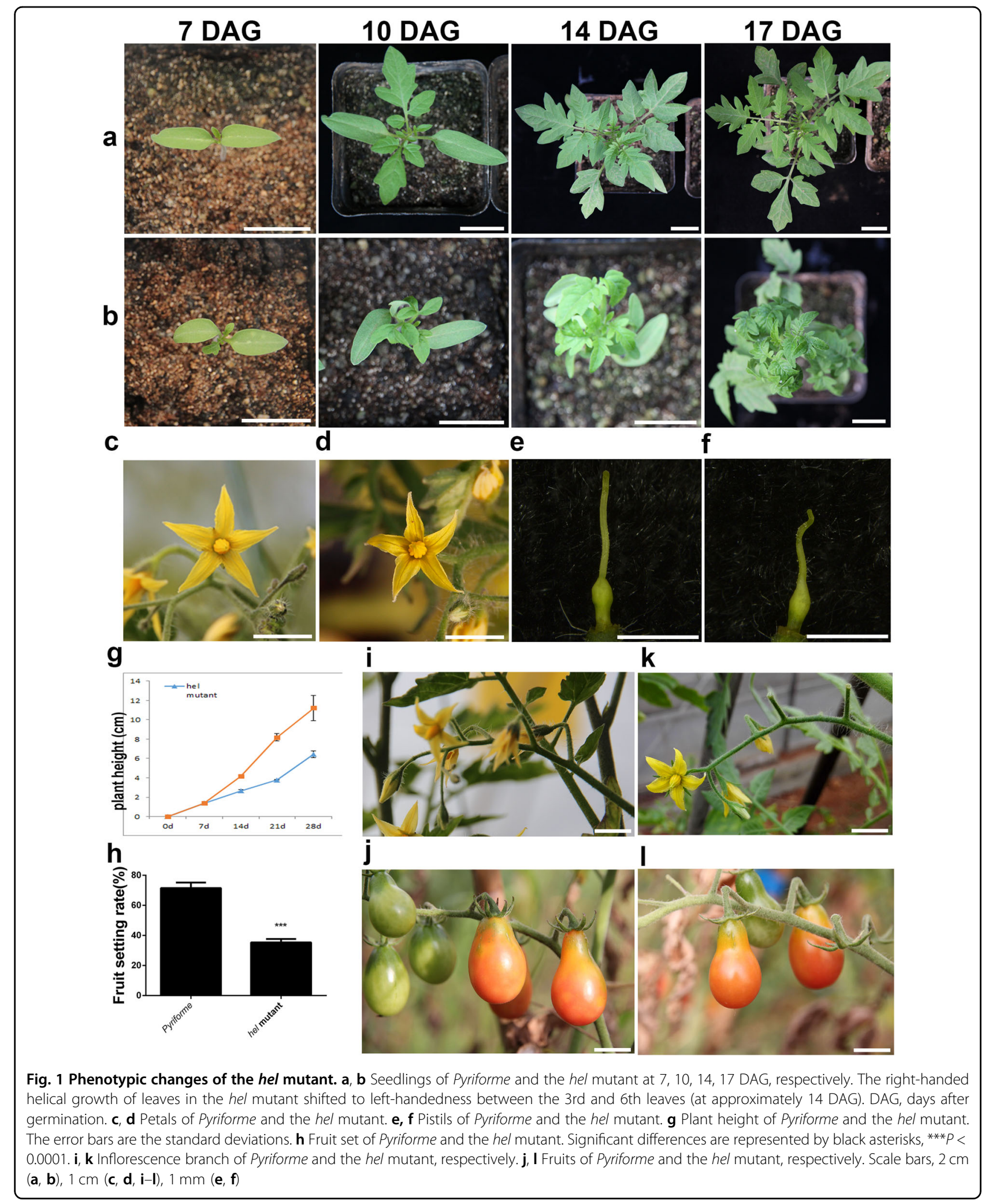

data from the N-pool and M-pool. 7.08 Gb of raw data were generated at an approximately $10 \times$ depth and with more than $99 \%$ coverage. After removing low-quality bases, 18,517,575 and 22,441,025 clean reads were obtained from the N-pool and M-pool, respectively. The average SNP index of SNPs located within a given 


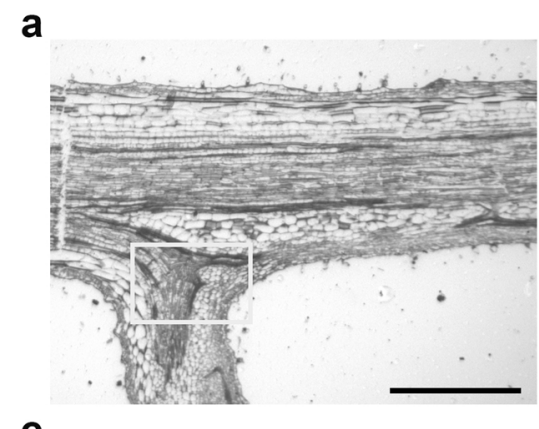

C

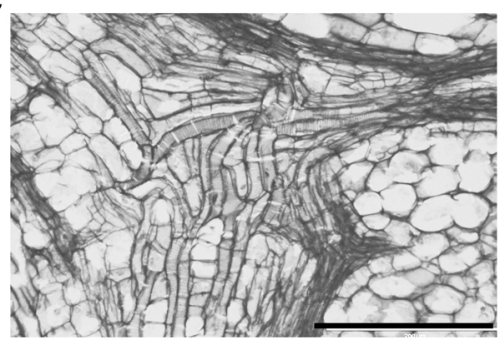

e

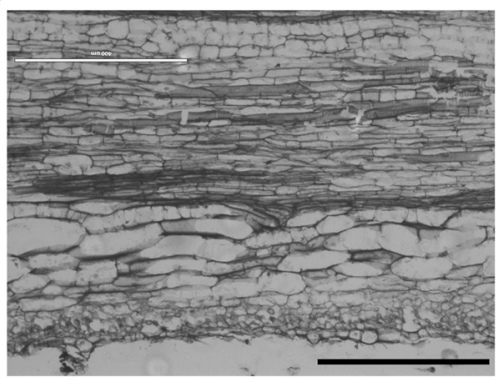

b

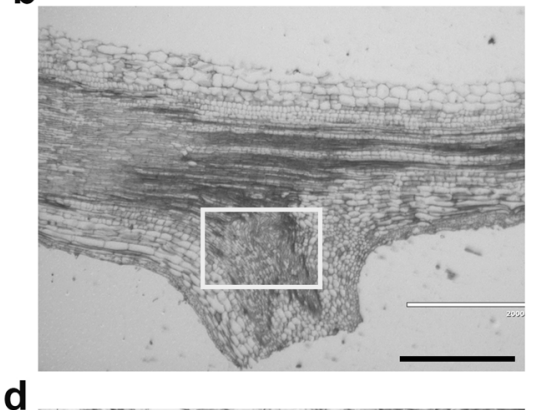

d

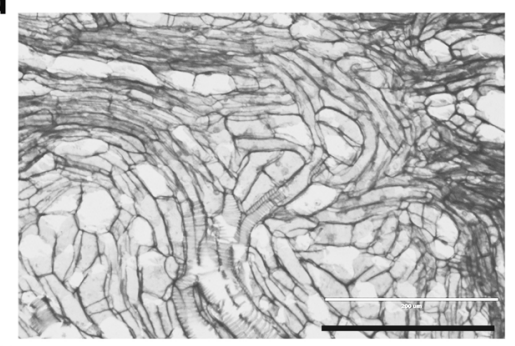

f

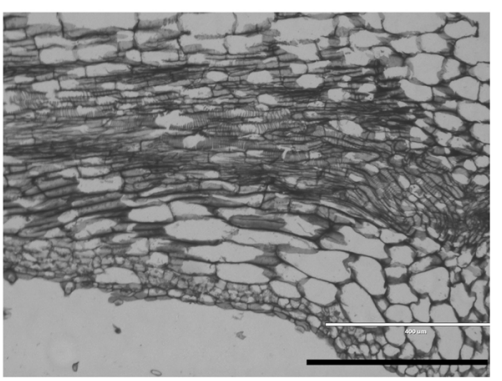

Fig. 2 Cytological observations of the hel mutant and its Pyriforme wild-type background. $\mathbf{a}, \mathbf{b}$ Transverse sections of the rachis of the first compound leaflet of Pyriforme and the hel mutant, respectively. The rectangle indicates the subsequent magnified area. $\mathbf{c}$, $\mathbf{d}$ Transverse sections of the petiolules of Pyriforme and the hel mutant, respectively. e, $\mathbf{f}$ Transverse sections of the rachises of the wild type and hel mutant. Scale bars, $1000 \mu \mathrm{m}(\mathbf{a}, \mathbf{b}), 200 \mu \mathrm{m}(\mathbf{c}, \mathbf{d}), 400 \mu \mathrm{m}(\mathbf{e}, \mathbf{f})$

genomic interval was then computed. According to the SNP index graph, the peak on chromosome 4 was significantly higher than the other peaks at the $95 \%$ significance level, suggesting that this locus may be responsible for the target phenotype (Fig. 3a). To further narrow the interval spanning $H E L$, we developed 6 molecular markers that were evenly distributed along $\mathrm{Chr}$. 4 (Fig. 3b, Supplementary Table S1). Genotyping of $108 \mathrm{~F}_{2}$ recessive individuals was performed with these polymorphic markers, and linkage analysis indicated that hel was delimited to the region between $\mathrm{CH} 4-25$ and CH4-35 at the terminal end of the long arm of Chr. 4, the loci of which were closely linked to hel with one and two recombination events, respectively (Fig. 3b).

We further identified nine recombinant individuals by screening 925 recessive $F_{2}$ individuals that possessed CH4-25 and CH4-35. We then used five molecular markers between CH4-25 and CH4-35: CH4-37, CAPS4-3, SNP4-2, SNP4-4, and SNP4-6 (Fig. 3c, Supplementary
Table S1). These nine recombinant individuals were genotyped using these newly developed markers (Supplementary Table S1). Finally, we delimited the $H E L$ gene to the region between SNP4-3 and SNP4-6, both of which were closely linked to the target gene with just one recombinant (Fig. 3c). SNP4-4 cosegregated with the HEL gene (Fig. 3c). Based on the tomato reference genome sequence, the physical distance between SNP4-3 and SNP4-4 was approximately $390 \mathrm{~kb}$ (Fig. 3c).

\section{Candidate genes of $H E L$}

Based on the tomato genome annotation (ITAG Release 4.0), the region between SNP4-2 and SNP4-6 encompassed 13 putative ORFs. (Fig. 3d, Table 1). Similar results were also revealed by analysis of the $\sim 390 \mathrm{~kb}$ sequence with FGENESH and GeneScan. The best hits of these ORFs include a myosin class II heavy chain protein, a C2 domain-containing protein-like protein, an aspartyl protease family protein, a metal ion-binding protein, a 


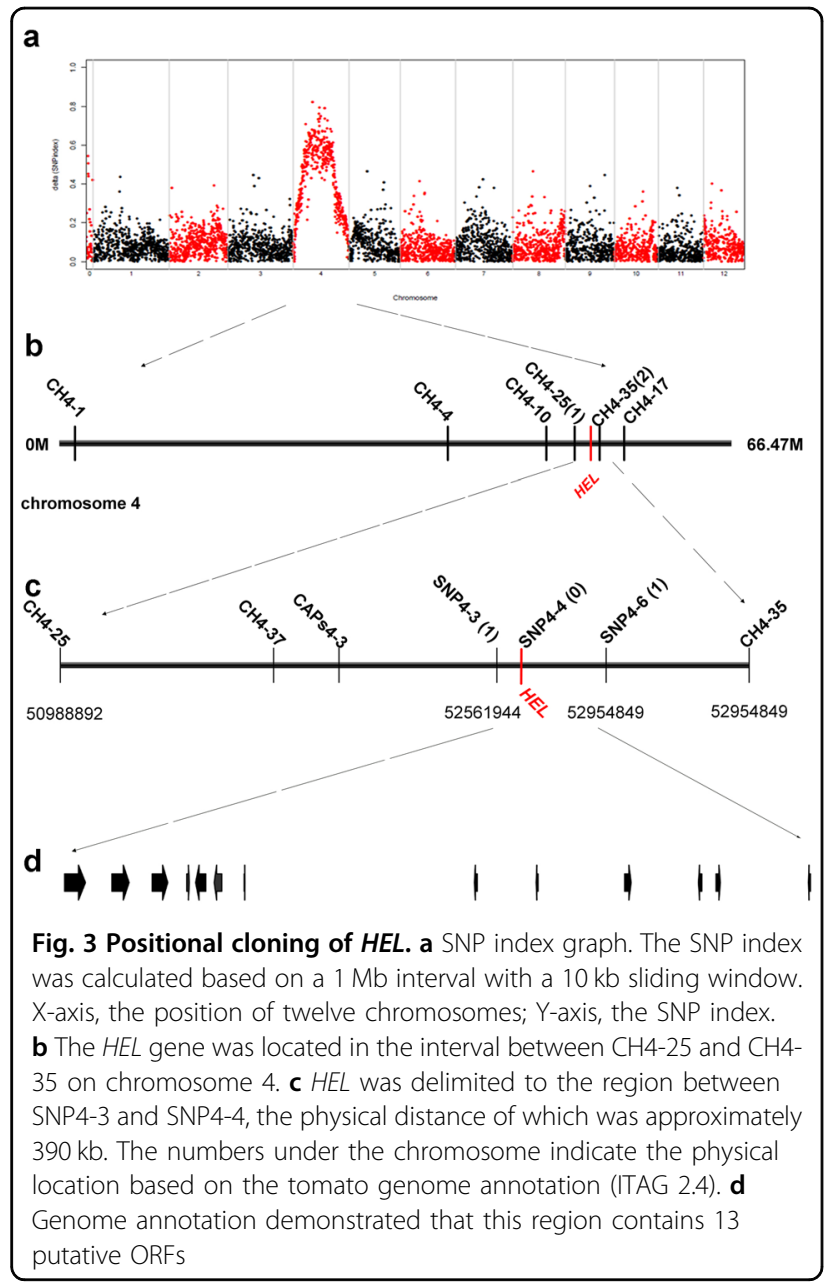

DNA-directed RNA polymerase I subunit RPA12 protein, a peptidyl-prolyl cis-trans isomerase protein, a ring finger protein, a laccase protein, etc.

Among these ORFs, ORF2 encodes a C2 domaincontaining protein-like protein, the homolog of which is essential for subtle right-handed torsion in Arabidopsis (Supplementary Fig. S2) ${ }^{21}$. In addition, ORF13 encodes a laccase protein, and mutation in its Arabidopsis homolog SKU5 gene caused a counterclockwise axial rotation bias $^{29}$. ORF2 and ORF13 were thus considered possible candidates for $H E L$. We amplified and sequenced the fulllength coding sequences and the putative promoter fragments of these two candidates from the hel mutant and its background material and identified a $27 \mathrm{bp}$ fragment replacement in the hel mutant (Fig. 4a). The fragment replacement resulted in a premature stop codon in ORF2 (Fig. 4b). Another candidate showed no sequence changes. These findings further supported the inference that ORF2 was the possible candidate responsible for the helical growth phenotype.

\section{The hel phenotype was complemented by ORF2 from Pyriforme}

To support that ORF2 is responsible for the target phenotype, we prepared a CRISPR/Cas9 vector and transformed this vector into Pyriforme via Agrobacteriummediated transformation. Two adjacent target sites were selected in the second exon of ORF2 (Fig. 4c). Editing of ORF2 in the CRISPR lines was detected via PCR and Sanger sequencing (Fig. 4d, e). The CRISPR line CR-ORF22 contained a $4 \mathrm{bp}$ insertion in the first target. The CRISPR

Table 1 Candidates in the region between SNP4-3 and SNP4-6

\begin{tabular}{|c|c|c|c|c|}
\hline ORF No. & Accession No. & Location(s) & Gene annotation & Length \\
\hline 1 & Solyc04g054470 & SL4.0ch04:51856797..51868225 & Myosin class II heavy chain & 11,427 \\
\hline 2 & Solyc04g054480 & SL4.0ch04:51882810..51892235 & C2 domain-containing protein-like & 9425 \\
\hline 3 & Solyc04g054490 & SL4.0ch04:51904985..51913563 & Aspartyl protease family protein & 8620 \\
\hline 4 & Solyc04g054500 & SL4.0ch04:51923982..51925276 & Metal ion-binding protein & 1295 \\
\hline 5 & Solyc04g054510 & SL4.0ch04:51934475..51928795 & DNA-directed RNA polymerase I & 5681 \\
\hline 6 & Solyc04g054520 & SL4.0ch04:51943221..51939111 & Peptidyl-prolyl cis-trans isomerase & 4111 \\
\hline 7 & Solyc04g054530 & SL4.0ch04:51955493..51955972 & Mutator-like transposase & 480 \\
\hline 8 & Solyc04g054570 & SL4.0ch04:52083688.52082085 & Unknown protein & 1603 \\
\hline 9 & Solyc04g054600 & SL4.0ch04:52117097..52116177 & Unknown protein & 920 \\
\hline 10 & Solyc04g054620 & SL4.0ch04:52164740..52168382 & Mutator-like transposase-like & 3643 \\
\hline 11 & Solyc04g054630 & SL4.0ch04:52207206..52205391 & Unknown protein & 1816 \\
\hline 12 & Solyc04g054650 & SL4.0ch04:52215059..52217431 & Ring finger protein 157 & 2383 \\
\hline 13 & Solyc04g054660 & SL4.0ch04:52266943..52265856 & Laccase & 1084 \\
\hline
\end{tabular}


a

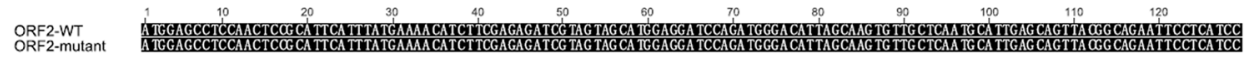

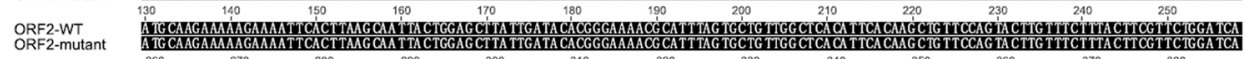

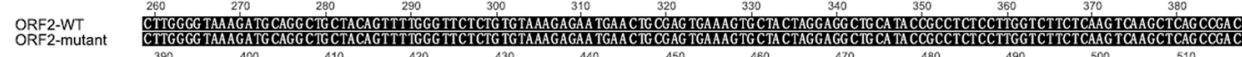

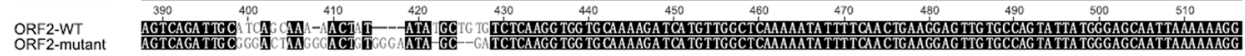

b
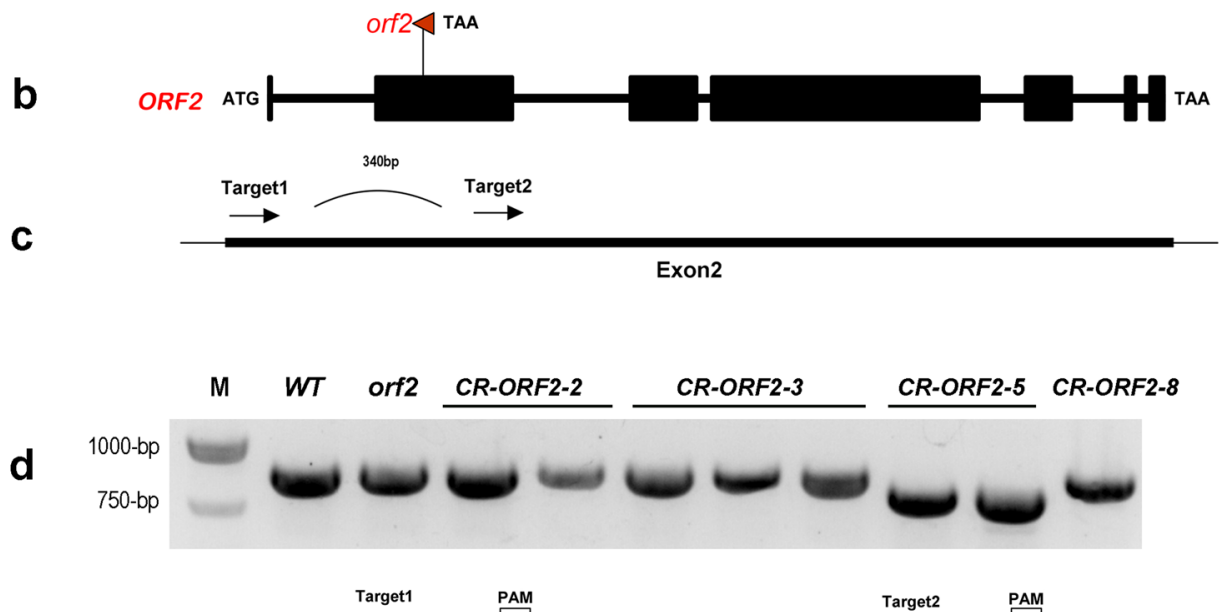

WT

AGTAGCATGGAGGATCCAGATGGGACATTAG...<322bp>...AAAAACTTATATGGTGTGTCTCAAGG TGG TGCAAAAGA

e

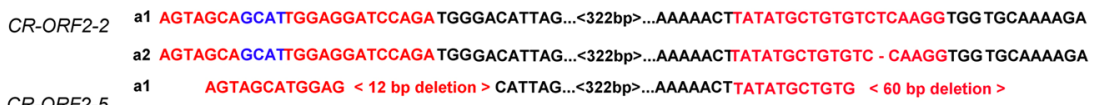

CR-ORF2-5

a2 AGTAGCATGGAGGATCCAGATGGGACATTAG...<322bp>...AAAAACTTATATGCTGTG <60 bp deletion >

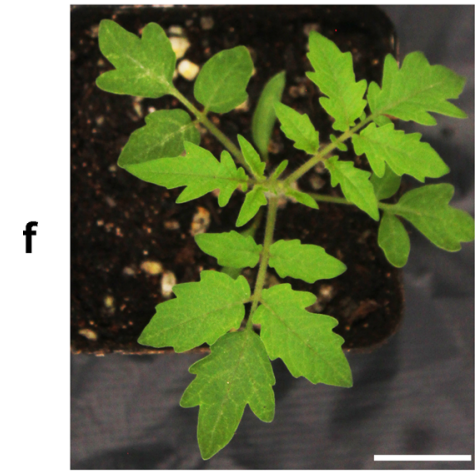

Pyriforme

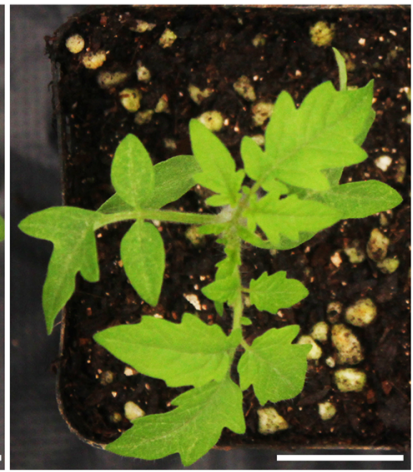

CR-ORF2-2 (a1)

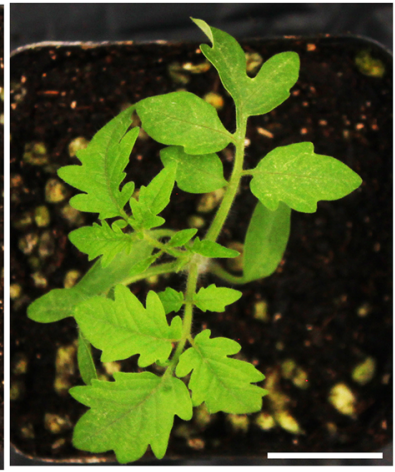

CR-ORF2-5 (a1)

Fig. 4 Sequence comparison of two alleles at the HEL locus and verification of transgenic ORF2 via the CRISPR-Cas9 system. a A 27 bp fragment replacement was detected between the hel mutant (ORF2-mutant) and wild-type Pyriforme (ORF2-WT). b Schematic illustration showing the premature stop codon in the second exon (the red triangle) of HEL in the hel mutant. c Two sgRNAs (arrows) targeting the second exon of ORF2. d PCR based identification of 4 CR-ORF2 T1 lines with a helical growth phenotype. M, marker; WT, wild type; hel, hel mutant; CR-ORF2-2 includes two alleles; CR-ORF2-5 includes two alleles, with a large fragment deletion. e Targeted mutations in CR-ORF2 transgenic plants. f Phenotypes of two ORF2 edited plants (CR-ORF2-2a1 and CR-ORF2-5a1). Scale bar, $2 \mathrm{~cm}$

line CR-ORF2-5a1 contained a 12 bp deletion downstream of the first target and a $60 \mathrm{bp}$ deletion downstream of the second target (Fig. 4d, e). These mutations in ORF2 resulted in a helical growth phenotype similar to that of the hel mutant (Fig. 4f). To further examine the biological function of ORF2, an overexpression construct for this candidate gene under the control of the CaMV-35S promoter was prepared and introduced into the hel mutant. Eight independent transformants were obtained. The helical growth phenotype of the hel mutant was complemented by the fragment from Pyriforme (Fig. 5a). QRTPCR analysis indicated that the expression level of ORF2 
a

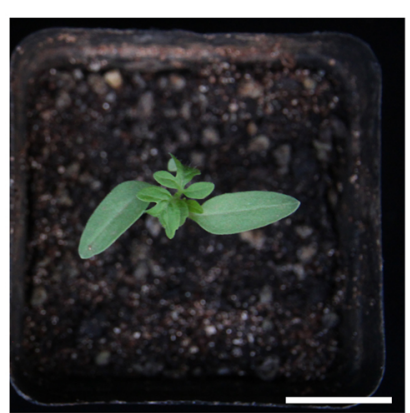

hel mutant

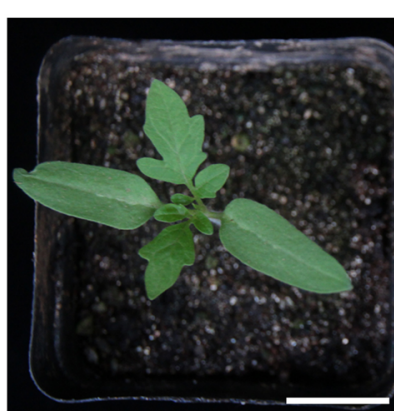

ORF2-OE-1

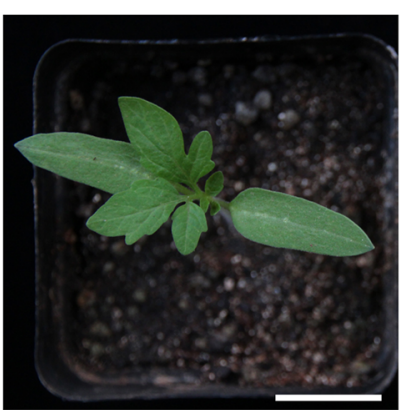

ORF2-OE-7
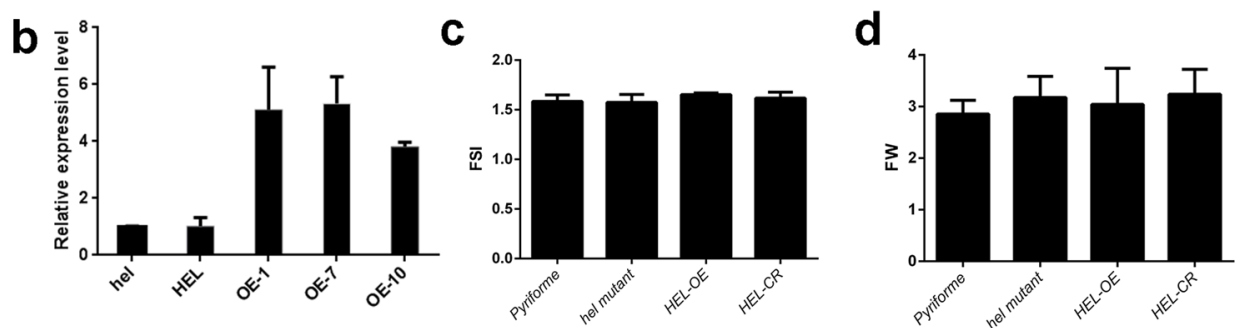

Fig. 5 Phenotypes of ORF2 overexpression lines. a The phenotype of the hel mutant was restored by ORF2 overexpression. $\mathbf{b}$ Expression level of ORF2 in the hel mutant, Pyriforme and overexpression lines. $\mathbf{c}$ and $\mathbf{d}$ The fruit shape index (FSI) and fruit weight (FW) of Pyriforme, the hel mutant, and ORF2 overexpression lines. Scale bar, $2 \mathrm{~cm}$

obviously increased in the transgenic plants (Fig. 5b). Taken together, these results suggest that ORF2 is the right candidate for $H E L$. Notably, the fruits of the transgenic plants showed no obvious differences compared to those of the hel mutant and Pyriforme (Fig. 5c, d).

\section{Discussion}

BSR-seq combined with molecular marker analysis is an efficient method for gene cloning

Bulked segregant analysis (BSA) has been extensively applied to identify molecular markers linked to a chromosomal region associated with a target phenotype ${ }^{30}$. Two bulk DNA samples collected from wild-type and mutant individuals separately need to be sequenced via whole-genome shotgun sequencing. Although the sequencing price has sharply dropped with rapid improvement of the technology underlying genome sequencing, this strategy is still not cost-effective in tomato. BSA in combination with RNA-seq was therefore developed as an efficient and cost-effective strategy for determining the chromosomal location of target genes $^{25,31-33}$. After the approximate region of the target gene was determined by the BSR-seq method, finemapping with molecular markers has been suggested to be feasible and effective in many crop species, such as wheat and cotton ${ }^{34-36}$. In this study, we first adopted the
BSR-seq method to identify the chromosomal location of the HEL gene. HEL was successfully mapped to chromosome 4 via a low-depth BSR-seq approach. Combining BSR-seq with classic marker analysis, we delimited HEL to within the region between markers SNP4-2 and SNP4-6. The efficiency of gene mapping with BSR-seq analysis and marker screening obviously improved.

\section{HEL possibly controls tomato helical growth via molecular mechanisms different from those in Arabidopsis}

In this study, we determined that HEL encodes a cellulose synthase interactive protein in tomato. There are $3 \mathrm{HEL}$ homologous genes in Arabidopsis: CSI1, CSI2 and CSI3. HEL shared $79 \%$ amino acid sequence identity with CSI1, $38.5 \%$ with CSI 2 and $44.5 \%$ with CSI3 (Fig. S2). CSI1 acts as a physical link between CESA complexes and cortical microtubules ${ }^{19,20,37}$. Previous investigations suggested that CSI1, CSI2, and CSI3 exhibited similar functions in guiding primary CSCs along CMTs during cellulose biosynthesis ${ }^{38}$. The orientation of cellulose microfibrils and cortical microtubules in mutants with mutations in the CSI1 gene was uncoupled $^{19,20}$. Furthermore, elongating roots, hypocotyls, and leaves of the csil mutant showed left-handed spiraling, whereas the inflorescence stems exhibited slightly right-handed spiraling twists ${ }^{20,21}$. Here, we found that the stems of the hel mutant exhibited left-handed twisting and 
vine-like growth after flowering; nevertheless, the leaves that initially showed right-handed helical growth became left-handed as the plants grew. However, similar phenotypes were not observed for the Arabidopsis csi1 mutant. Therefore, it can be speculated that the molecular mechanism mediated by $H E L$ underlying helical growth of tomato is different from that of Arabidopsis. In tomato, the corkscrew (crs) mutant also exhibited helical growth ${ }^{39}$. However, the phenotypic details of cotyledons, leaves, stems, and anther cones of the crs mutant differed from those of the hel mutant. It would be worthwhile to clone the CRS gene and determine the possible roles of this gene in helical growth of tomato.

\section{HEL may work together with MAPs during helical growth formation}

The microtubule-microfibril alignment model laid a theoretical foundation for the helical growth model. It is still not clear why mutations in highly conserved proteins such as HEL and CSI1 cause opposite phenotypes. The different directions of helical growth of Arabidopsis are caused by a series of mutations in tubulins or MAPs ${ }^{5-10,29,40,41}$. CSI1 plays an important role in sustaining CSC movement along CMTs. Moreover, the movement direction is dependent on other MAPs, such as kinesins ${ }^{40}$. As expected, mutations in kinesin resulted in right-handed helical root growth ${ }^{42}$. Hence, it can be deduced that the microtubule-microfibril alignment model is governed by complex variable systems that can be mediated by multiple protein complexes. These different combinations of proteins could lead to different phenotypes. In this study, the fruits of Pyriforme exhibited a pear shape, similar to those of the ovate mutant. OVATE has been proven to play an essential role in microtubule arrangement by interacting with TON1 Recruiting Motif (TRM) proteins ${ }^{43,44}$. Moreover, the stems of the hel mutant showed no helical growth at the early stage, which gradually shifted to left-handed twisting after flowering. This shift was closely related to the expression level of OVATE ${ }^{45}$. We thus infer that these MAPs may be involved in the regulation of tomato helical growth by interacting with HEL.

\section{Application of CRISPR/Cas9 efficiently restructured the erect architecture of tomato to a helical architecture}

Recently, the CRISPR/Cas9 strategy has been successfully used to reprogram the vine-like structure of plants into a compact stature ${ }^{46,47}$. Both HEL and CSI1 are essential for helical growth of tomato and Arabidopsis, suggesting that members of this family can be edited to alter the growth habit of other plant species. In the present study, we mutagenized HEL using the CRISPR/ Cas9 system and obtained several mutant lines. Compared with those of erect tomato plants, the stems of the mutated lines showed a left-handed helix and twined around a support under artificial traction, which can help save time required for winding onto a support and the amount of ties and ropes. The inflorescence branches and fruits of the hel mutant showed no obvious differences compared to those of wild-type plants. Although the tomato fruit set of the hel mutant decreased, elucidating the molecular basis underlying tomato helical growth may provide a new idea for formulating modern sustainable agricultural practices in the future.

\section{Acknowledgements \\ This work was supported by grants from the NSFC (31672149, 31991182 and 31872122) and the National Key R\&D Program of China (2017YFD0101902).}

Conflict of interest

All the authors declare that they have no conflicts of interest.

Supplementary Information accompanies this paper at (https://doi.org/ 10.1038/s41438-020-00402-0).

Received: 10 May 2020 Revised: 21 July 2020 Accepted: 27 July 2020 Published online: 01 November 2020

\section{References}

1. Janoria M. Basic plant ideotype for rice. International Rice Research Newsletter (Philippines) 1989

2. Steeves T., Sussex I. Pattern in plant development, vol. 101 (Cambridge University Press, 1989).

3. Jaffe, M. J. \& Galston, A. W. The physiology of tendrils. Minn. Med 19, 417-434 (2003).

4. Smyth, D. R. Helical growth in plant organs: mechanisms and significance. Development 143, 3272-3282 (2016).

5. Furutani, I., Watanabe, Y., Prieto, R., Masukawa, M. \& Hashimoto, T. The SPIRAL genes are required for directional control of cell elongation in Arabidopsis thaliana. Development 127, 4443-4453 (2000).

6. Thitamadee, S., Tuchihara, K. \& Hashimoto, T. Microtubule basis for left-handed helical growth in Arabidopsis. Nature 417, 193-196 (2002).

7. Nakajima, K., Furutani, I., Tachimoto, H., Matsubara, H. \& Hashimoto, T. SPIRAL1 encodes a plant-specific microtubule-localized protein required for directional control of rapidly expanding Arabidopsis cells. Plant Cell 16, 1178-1190 (2004).

8. Sedbrook, J. C., Ehrhardt, D. W., Fisher, S. E., Scheible, W. R. \& Somerville, C. R. The Arabidopsis SKU6/SPIRAL1 gene encodes a plus end-localized microtubule-interacting protein involved in directional cell expansion. Plant Cell 16, 1506-1520 (2004)

9. Yao, M., Wakamatsu, Y., Itoh, T. J., Shoji, T. \& Hashimoto, T. Arabidopsis SPIRAL2 promotes uninterrupted microtubule growth by suppressing the pause state of microtubule dynamics. J. Cell Sci. 121, 2372-2381 (2008).

10. Wightman, R., Chomicki, G., Kumar, M., Carr, P. \& Turner, S. R. SPIRAL2 determines plant microtubule organization by modulating microtubule severing. Curr. Biol. 23, 1902-1907 (2013).

11. Ishida, T., Kaneko, Y., Iwano, M. \& Hashimoto, T. Helical microtubule arrays in a collection of twisting tubulin mutants of Arabidopsis thaliana. Proc. Natl Acad. Sci. USA 104, 8544-8549 (2007).

12. Nakamura, M. \& Hashimoto, T. A mutation in the Arabidopsis -tubulin-containing complex causes helical growth and abnormal microtubule branching. J. Cell Sci. 122, 2208-2217 (2009).

13. Hashimoto, T. Dissecting the cellular functions of plant microtubules using mutant tubulins. Cytoskeleton 70, 191-200 (2013).

14. Hardham, A. R. \& Gunning, B. E. Structure of cortical microtubule arrays in plant cells. J. Cell Biol. 77, 14-34 (1978).

15. Delmer, D. P. \& Amor, Y. Cellulose biosynthesis. Plant Cell 7, 987-1000 (1995).

16. Arioli, T. et al. Molecular analysis of cellulose biosynthesis in Arabidopsis. Science 279, 717-720 (1998).

17. Baskin, T. I. Anisotropic expansion of the plant cell wall. Annu Rev. Cell Dev. Biol. 21, 203-222 (2005).

18. $\mathrm{Gu}, \mathrm{Y}$. et al. Identification of a cellulose synthase-associated protein required for cellulose biosynthesis. Proc. Natl Acad. Sci. USA 107, 12866-12871 (2010). 
19. Li, S., Lei, L., Somenville, C. R. \& Gu, Y. Cellulose synthase interactive protein 1 (CSI1) links microtubules and cellulose synthase complexes. Proc. Natl Acad. Sci. USA 109, 185-190 (2012).

20. Bringmann, $M$. et al. POM-POM2/CELLULOSE SYNTHASE INTERACTING1 is essential for the functional association of cellulose synthase and microtubules in Arabidopsis. Plant Cell 24, 163-177 (2012).

21. Landrein, B. et al. Impaired cellulose synthase guidance leads to stem torsion and twists phyllotactic patterns in Arabidopsis. Curr. Biol. 23, 895-900 (2013)

22. Bradley, D., Ratcliffe, O., Vincent, C., Carpenter, R. \& Coen, E. Inflorescence commitment and architecture in Arabidopsis. Science 275, 80-83 (1997).

23. Pnueli, L. The SELF-PRUNING gene of tomato regulates vegetative to reproductive switching of sympodial meristems and is the ortholog of CEN and TFL1. Development 125, 1979-1989 (1998).

24. Li, H. \& Durbin, R. Fast and accurate short read alignment with BurrowsWheeler transform. Bioinformatics 25, 1754-1760 (2009).

25. Liu, S., Yeh, C. T., Tang, H. M., Dan, N. \& Schnable, P. S. Gene mapping via bulked segregant RNA-Seq (BSR-Seq). PloS ONE 7, e36406 (2012).

26. Fulton, T. M., Chunwongse, J. \& Tanksley, S. D. Microprep protocol for extraction of DNA from tomato and other herbaceous plants. Plant Mol. Biol. Rep. 13, 207-209 (1995).

27. Lander, E. S. et al. MAPMAKER: an interactive computer package for constructing primary genetic linkage maps of experimental and natural populations. Genomics 1, 174-181 (1987).

28. Xiao, J. et al. Dissection of GA 20-oxidase members affecting tomato morphology by RNAi-mediated silencing. Plant Growth Regul. 50, 179-189 (2006).

29. Sedbrook, J. C., Carroll, K. L., Hung, K. F., Masson, P. H. \& Somerville, C. R. The Arabidopsis SKU5 gene encodes an extracellular glycosyl phosphatidylinositol-anchored glycoprotein involved in directional root growth. Plant Cell 14, 1635-1648 (2002).

30. Michelmore, R. W., Paran, I. \& Kesseli, R. V. Identification of markers linked to disease-resistance genes by bulked segregant analysis - a rapid method to detect markers in specific genomic regions by using segregating populations. Proc. Natl Acad. Sci. USA 88, 9828-9832 (1991).

31. Wang, R. et al. Bulk segregant RNA-seq reveals expression and positional candidate genes and allele-specific expression for disease resistance against enteric septicemia of catfish. Bmc Genomics 14, 929 (2013).

32. Kim, S., Kim, C.-W., Park, M. \& Choi, D. Identification of candidate genes associated with fertility restoration of cytoplasmic male-sterility in onion (Allium cepa L.) using a combination of bulked segregant analysis and RNAseq. Theor. Appl. Genet. 128, 2289-2299 (2015).
33. Illa-Berenguer, E., Van Houten, J., Huang, Z. \& van der Knaap, E. Rapid and reliable identification of tomato fruit weight and locule number loci by QTLseq. Theor. Appl. Genet. 128, 1329-1342 (2015).

34. Ramirez-Gonzalez, R. H. et al. RNA-Seq bulked segregant analysis enables the identification of high-resolution genetic markers for breeding in hexaploid wheat. Plant Biotechnol. J. 13, 613-624 (2015).

35. Li, L. et al. A novel nitrogen-dependent gene associates with the lesion mimic trait in wheat. Theor. Appl. Genet. 129, 2075-2084 (2016).

36. Thyssen, G. N. et al. Next generation genetic mapping of the Ligon-lintless-2 (Li2) locus in upland cotton (Gossypium hirsutum L.). Theor. Appl. Genet. 127 2183-2192 (2014)

37. Mei, Y., Gao, H. B., Yuan, M. \& Xue, H. W. The Arabidopsis ARCP protein, CSI1, which is required for microtubule stability, is necessary for root and anther development. Plant Cell 24, 1066-1080 (2012).

38. Lei, L., Li, S., Du, J., Bashline, L. \& Gu, Y. CELLULOSE SYNTHASE INTERACTIVE3 regulates cellulose biosynthesis in both a microtubule-dependent and microtubule-independent manner in Arabidopsis. Plant Cell 25, 4912-4923 (2013).

39. Jáquez-Gutiérrez, M. et al. Phenotypic and genetic characterization of tomato mutants provides new insights into leaf development and its relationship to agronomic traits. BMC Plant Biol. 19, 1-24. (2019).

40. Hamada, T. Microtubule organization and microtubule-associated proteins in plant cells. Int. Rev. Cell Mol. Biol. 312C, 1-52 (2014).

41. Struk, S. \& Dhonukshe, P. MAPs: cellular navigators for microtubule array orientations in Arabidopsis. Plant Cell Rep. 33, 1-21 (2014).

42. Sakai, T. et al. Armadillo repeat-containing kinesins and a NIMA-related kinase are required for epidermal-cell morphogenesis in Arabidopsis. Plant J. 53, 157-171 (2008).

43. Wu, S. et al. A common genetic mechanism underlies morphological diversity in fruits and other plant organs. Nat. Commun. 9, 4734 (2018).

44. Spinner, L. et al. A protein phosphatase $2 A$ complex spatially controls plant cell division. Nat. Commun. 4, 1863 (2013).

45. Liu, J., Van Eck, J., Cong, B. \& Tanksley, S. D. A new class of regulatory genes underlying the cause of pear-shaped tomato fruit. Proc. Natl Acad. Sci. USA 99, 13302-13306 (2002).

46. Varkonyi-Gasic, E. et al. Mutagenesis of kiwifruit CENTRORADIALIS-like genes transforms a climbing woody perennial with long juvenility and axillary flowering into a compact plant with rapid terminal flowering. Plant Biotechnol. J. 17, 869-880 (2019).

47. Kwon, C.-T. et al. Rapid customization of Solanaceae fruit crops for urban agriculture. Nat. Biotechnol. 38, 182-188 (2019). 